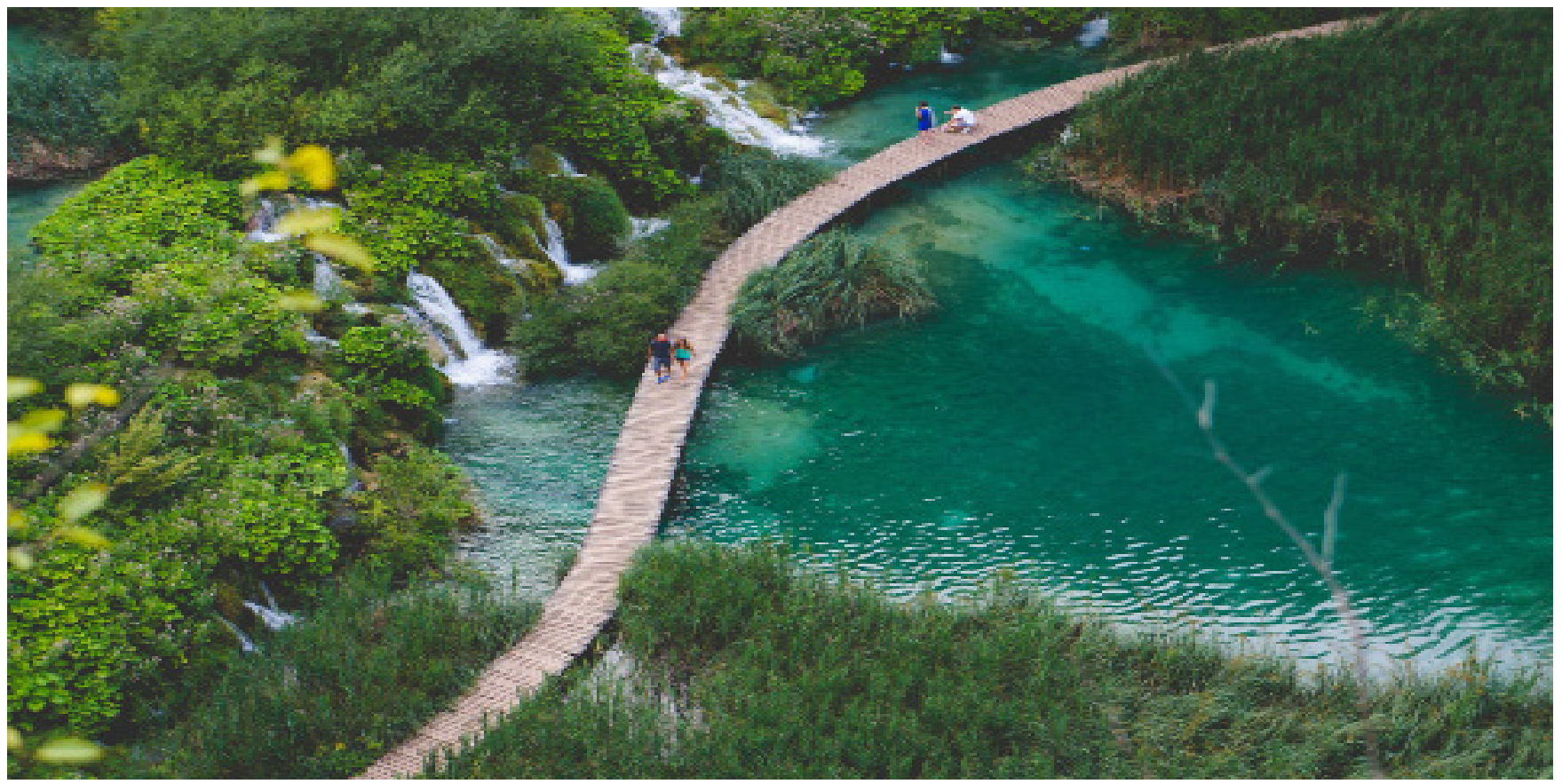

\title{
Agroturismo una nueva alternativa para impulsar el desarrollo económico en el municipio de Ocaña
}

\section{Agroturism a new alternative to promote economic development in the municipality of Ocaña}

Johann Fernando Hoyos-Patiño

Magister en Sistemas Sostenibles de Producción,

jfhoyosp@ufpso.edu.co, https://orcid.org/0000-0002-0377-4664

Universidad Francisco de Paula Santander, Ocaña, Colombia.

Daniel Antonio Hernández-Villamizar

Magister en Ciencias Agrarias con mención en Producción Animal, dahernadezv@ufpso.edu.co, https://orcid.org/0000-0003-1971-8365,

Universidad Francisco de Paula Santander, Ocaña, Colombia.

Blanca Liliana Velásquez-Carrascal

Magister en Economía, bl_velasquez@fesc.edu.co

https://orcid.org/0000-0001-7718-853X Fundación de Estudios Superiores

Comfanorte - FESC, Cúcuta, Colombia. 


\section{4}

Recibido: Junio 13 de 2018

Aceptado: Diciembre 3 de 2018

Cómo citar: J.F Hoyos-Patiño, D.A Hernández-Villamizar y B.L Velásquez-Carrascal, “Agroturismo una nueva alternativa para impulsar el desarrollo económico en el municipio de Ocaña", Sostenibilidad, Tecnología y Humanismo, vol. 10, no. 1, 63-69, 2019.

Resumen

El agroturismo funciona como motor económico en las regiones, generando impactos positivos, convirtiéndose en instrumento de difusión cultural y desarrollo. Con adecuada capacitación y organización, los productores pueden encontrar en esta actividad nuevas alternativas dediversificación parasus procesos productivos. La finalidad de esta investigación, fue elaborar una propuesta agroturística en el municipio de Ocaña-Norte de Santander, para impulsar el potencial paisajístico y productivo de la zona, bajo las condiciones de pequeños y medianos productores. Caracterizando la posible ruta agroturística con la ayuda de una matriz DOFA. En los resultados se proyecta una propuesta agroturística, que consiste en describir los procesos productivos y de servicios que pueden prestar los sistemas de producción, enfocados a turistas regionales, nacionales e internacionales. Por otra parte, se contemplan una serie de rutas turísticas adicionales que complementan los atractivos de la zona. Igualmente, se plantean algunas estrategias que faciliten el montaje del modelo agroturístico en el municipio de Ocaña, modelo de capacitación a los productores en aspectos como: gastronomía, contabilidad y servicio al turista. Se finaliza el estudio, resaltando el potencial de esta actividad económica, para la generación de ingresos no convencionales para los productores.

Palabras clave: Agroturismo, alternativa, productor agropecuario, sistema de producción, desarrollo económico
Abstract

Agrotourism works as economic engine in the regions, generating positive impacts, becoming an instrument of cultural diffusion and development. With adequate training and organization, farmer can find in this activity new diversification alternatives for their production processes. The purpose of this research was to develop an agrotourism proposal in the municipality of Ocaña-Norte de Santander, to boost the landscape and productive potential of the area, under the conditions of small and medium producers. Characterizing the possible agrotourism route with the help of a SWOT matrix. In the results, an agrotourism proposal is projected, which consists in describing the production and service processes that can be provided by production systems, focused on regional, national and international tourists. On the other hand, a series of additional tourist routes that complement the attractions of the area are contemplated. Likewise, some strategies are proposed that facilitate the assembly of the agrotourist model in the municipality of Ocaña, a training model for producers in aspects such as: gastronomy, accounting and tourist service. The study is completed, highlighting the potential of this economic activity, for the generation of unconventional income for producers.

Keywords: $\quad$ Agrotourism, alternative, agricultural producer, production system, economic development 
Introducción

Desde hace unas décadas se ha observado el creciente interés por el turismo de agricultura, ya sea por descanso o por querer tener una conexión más cercana con la naturaleza o con las costumbres de una localidad [4]. A nivel mundial se evidencia una demanda de este tipo de turismo; países como España, Panamá, Chile y México le han apostado a dicha actividad empleos [1], como actividad emergente y oportunidad de desarrollo [2]; debido a que han visto que algunos turistas están interesados en vivir la experiencia de cultivar la tierra [3].

A lo largo de los últimos años se ha creado una tendencia mundial al cuidado, protección y apreciación de la naturaleza, actualmente se puesta por una nueva tendencia turística, donde el turista está más interesado en vivenciar experiencias ecológicas, ya no quiere estar en un lugar lleno de gente como las capitales más importantes del mundo o las playas más frecuentadas; buscando así, ambientes más tranquilo, relajado y libre de cualquier estrés [4].

Últimamente en países como Suecia, Austria, Alemania, Holanda, Italia o Francia; muestran un interés por el agroturismo ya que este se ha incrementado en la última década. Según [5] España es un claro ejemplo en cuanto a fomentar este tipo de turismo; ya que cuenta con varias extensiones de campo e incluso ya hay granjas con alojamiento que le brindan toda la experiencia completa de la vida del campo, como por ejemplo cosechar alimentos, ordeñar vacas, dar de comer a los animales, entre otras actividades propias de los procesos productivos [6].

Así mismo en Latinoamérica también se ha inclinado por apoyar e impulsar más a este tipo de turismo, según [7] países como Chile, Argentina, Colombia, Perú y Costa
Rica; han decidido explotarlo. Costa Rica es un buen ejemplo de agroturismo, es un país pequeño en su extensión, pero esto no evita el poder desarrollar esta actividad, el tipo de agroturismo que ellos impulsan en la zona caribe y sur, en las zonas indígenas. El país de Argentina con su amplio territorio desarrolla su agroturismo mediante actividades como el cultivo y producción de viñedos, este país también es famoso por su ganado de calidad y lugares naturales como lagos, bosque y demás [3].

Con referencia a lo anterior, se puede decir que Colombia es un país con una amplia oportunidad para poder desarrollar el agroturismo, ya que cuenta con una gran variedad de flora y fauna. Actualmente alrededor del país hay varios departamentos que han trabajado este tipo de turismo; abriendo las puertas de sistemas de producción o fincas a turistas nacionales e internacionales para que ellos puedan experimentar y valorar más el trabajo del productor rural; los cuales ayudan a la economía del país y así mismo tener una mirada más profunda de las tradiciones y cultura [8].

Colombia es un país privilegiado por sus riquezas naturales, belleza geográfica, diversidad de ecosistemas y climas [9], todo esto lo convierte en un lugar idóneo para que las personas se conecten con la naturaleza. Se debe lograr que los productores conozcan las oportunidades que les abre esta forma de hacer turismo, enseñando el día a día del funcionamiento de los procesos productivos.

Colombia es considerado el segundo país más biodiverso del mundo, al ser una región tropical cuenta con una gran variedad de especies de animales y plantas, haciendo de esto un gran atractivo natural para los visitantes [3]. Por esta razón se puede concluir que el agroturismo se ha convertido en una nueva manera de fomentar el desarrollo de un 


\section{6}

país o una localidad, por lo tanto, se le apuesta a fomentar esta práctica en el municipio de Ocaña, Norte de Santander podría darse un gran crecimiento a su economía si se pudiera implementar este tipo de turismo; no solo se le aporta al sector económico sino también a impulsar las empresas agropecuarias de la región. Debido a esto surge la pregunta, ¿es viable impulsar el agroturismo en el municipio de Ocaña?

El propósito de la investigación fue analizar la viabilidad del agroturismo en el municipio de Ocaña, como una actividad económica que genera ingresos, destacando el valor del campo; asegurando la conservación de manera segura y sustentable, encontrando una manera adecuada de aprovechar este turismo de la mano con el sector agropecuario.

Materiales y métodos

La investigación se enfocó en estudiar las posibles oportunidades para impulsar el agroturismo en el municipio de Ocaña, Norte de Santander; ya que debido a la creciente demanda observada por este tipo de turismo rural en países como España [5], observandola posibilidad de ayudar al sector agropecuario e impulsar la economía de la localidad de Ocaña.

La Metodología desarrollada para el presente trabajo de investigación es documental de tipo exploratorio descriptivo.

El trabajo se realizó con ayuda de estudiantes del programa de Zootecnia de la Universidad Francisco de Paula Santander sede Ocaña y estudiantes de Administración Turística y Hotelera de la Fundación de Estudios Superiores Comfanorte - FESC, recopilando material bibliográfico que apoye la hipótesis de potencialidad agroturística que tiene Ocaña.
Para respaldar la investigación se consultaron fuentes secundarias de información (revistas, artículos y fuentes electrónicas).

Para ello se identificaron, caracterizaron y analizaron dos dimensiones (actividades propias de los sistemas de producción y zonas con potencial turístico) primera variable de estudio.

El agroturismo, se consideró como (causa) variable independiente y la competitividad, (consecuencia) como variable dependiente, la cual otorga desarrollo económico a la región.

Resultados

Los procesos agropecuarios por décadas se han posicionado como la principal actividad productiva desarrollada en la provincia de Ocaña.

En la actualidad, la falta de políticas claras y de actores políticos comprometidos con el desarrollo integral de la región, han diezmado el crecimiento agropecuario como fuente de ingresos y desarrollo para la población rural.

\section{Análisis DOFA}

Para obtener un panorama general, se sintetiza en una matriz DOFA, algunas de las características principales que permitieron dimensionar el estado actual de la zona y contextualizarla en materia de competitividad, para la creación de la ruta agroturística del municipio de Ocaña (Tabla 1). 
Tabla 1. Matriz DOFA municipio de Ocaña en relación al potencial agroturístico

\begin{tabular}{|c|c|}
\hline Fortalezas & Debilidades \\
\hline Posibilidad de ofrecer servicios durante todo el año & \multirow{5}{*}{$\begin{array}{l}\text { Falta de capacitación en atención turistica, gastronomía, } \\
\text { manejo financiero y contable de los procesos. } \\
\text { Infraestructura de atención, de servicios y logísticos } \\
\text { inexistentes o en proceso de implementación } \\
\text { Identidad rural no definida. } \\
\text { Falta de organización y de canales de comunicación } \\
\text { entre productores agropecuarios y entidades } \\
\text { gubernamentales para el desarrollo del sector. }\end{array}$} \\
\hline $\begin{array}{l}\text { Paso obligado por tierra a turistas con destino al } \\
\text { departamento del Cesar y la Costa Atlántica. }\end{array}$ & \\
\hline Diversidad de atractivos turísticos tradicionales & \\
\hline $\begin{array}{l}\text { Gran arraigo en la zona desarrollando labores } \\
\text { agropecuarios }\end{array}$ & \\
\hline $\begin{array}{l}\text { La implementación de procesos agroturístico, se } \\
\text { presenta como alternativa factible para apoyar el } \\
\text { desarrollo de la región }\end{array}$ & \\
\hline Oportunidades & Amenazas \\
\hline $\begin{array}{l}\text { Aumentar los ingresos de los productores agropecuarios, } \\
\text { al participar en actividades agroturísticas. }\end{array}$ & $\begin{array}{l}\text { Resistencia del sector empresarial para el apoyo de } \\
\text { emprendimientos agroturísticos. }\end{array}$ \\
\hline $\begin{array}{l}\text { Comercializar y posicionar como productos orgánicos } \\
\text { y/o ecológicos obtenidos en las fincas. }\end{array}$ & $\begin{array}{l}\text { Conflicto de intereses político administrativo entre } \\
\text { actores del proceso. }\end{array}$ \\
\hline $\begin{array}{l}\text { Apoyar en los procesos académicos para formación de } \\
\text { nuevos profesionales con perfil en emprendimiento. }\end{array}$ & $\begin{array}{l}\text { Carencia de propuestas y soluciones sólidas que brinde } \\
\text { seguridad para la población. }\end{array}$ \\
\hline $\begin{array}{l}\text { Mejorar y abrir nuevos canales comerciales para los } \\
\text { sistemas de producción, que permitan fortalecer el } \\
\text { mercado regional, departamental y nacional }\end{array}$ & $\begin{array}{l}\text { Desinterés de los entes gubernamentales en general para } \\
\text { el desarrollo turístico y agroturístico de la región. }\end{array}$ \\
\hline $\begin{array}{l}\text { Ayudar en el fortalecimiento de la propuesta de clúster } \mathrm{T} \\
\text { turístico del municipio de Ocaña. }\end{array}$ & $\begin{array}{l}\text { Efecto negativo al afrontar la competencia creada por el } \\
\text { desarrollo turístico del sur del departamento del Cesar. }\end{array}$ \\
\hline
\end{tabular}

Al analizar las características de la matriz DOFA, se obtuvieron los objetivos estratégicos para la propuesta de la ruta agroturística (Tabla 2).

Tabla 2. Objetivos estratégicos para el desarrollo agroturístico de Ocaña.

\begin{tabular}{|c|c|}
\hline Potencialidades: (Fortalezas-0portunidades) & Riesgos: (Fortalezas-Amenazas) \\
\hline $\begin{array}{l}\text { Aprovechar la amplia variedad de sistemas de } \\
\text { producción agropecuarios con atractivos } \\
\text { agroturísticos, para dar a conocer la zona a nivel } \\
\text { nacional e internacional. }\end{array}$ & $\begin{array}{l}\text { Aprovechar el apoyo en formación empresarial y } \\
\text { emprendedora por parte de la Universidad Francisco de } \\
\text { Paula Santander sede Ocaña UFPSO y la Fundación de } \\
\text { Estudios Superiores COMFANORTE - FESC, para } \\
\text { enfrentar los riesgos financieros y de competencia en el } \\
\text { sector. }\end{array}$ \\
\hline $\begin{array}{l}\text { Aprovechar la amplia experiencia en procesos } \\
\text { productivos agropecuarios, que creen nuevos ingresos } \\
\text { para los productores y la región. }\end{array}$ & $\begin{array}{l}\text { terrestre obligado de turistas, debe aprovecharse } \\
\text { otivar en los productores involucrados la ofertar } \\
\text { os competitivos y de excelente calidad. }\end{array}$ \\
\hline Desafios: (Debilidades-0portunidades) & Limi \\
\hline $\begin{array}{l}\text { Promover el mejoramiento de los canales de } \\
\text { comunicación y organización entre productores- } \\
\text { gobierno-academia, que propendan en el } \\
\text { mejoramiento del nivel de vida de los productores } \\
\text { participantes en agroturismo. } \\
\text { Implementar y adecuar la infraestructura necesaria } \\
\text { para el desarrollo de actividades comerciales para la } \\
\text { venta, buscando posicionar en el mercado los } \\
\text { productos orgánicos y/o ecológicos elaborados. }\end{array}$ & $\begin{array}{l}\text { ión turística, gastronomía, } \\
\text { le de los procesos, se debe } \\
\text { ejorar la oferta del servicio } \\
\text { entas a los productores que } \\
\text { nto, para fortalecer y mejorar } \\
\text { on las empresas de viajes y } \\
\text { ervicio. }\end{array}$ \\
\hline
\end{tabular}

Para la implementación de un proceso agroturístico en el municipio de Ocaña, se requiere la participación activa del productor agropecuario en actividades comerciales y de servicios. Para lograr estas metas la implementación se divide en dos fases de ejecución:

Fase 1. Desarrollar una diversificación de las actividades al interior del sistema de producción.

Fase2. Fortalecerunaserie deatraccionesy rutasturísticas adicionales, fuera de las instalaciones de los sistemas de producción, que permitan no solo la venda del concepto del sistema de producción como elemento agroturístico, sino la idea de región como marca turística. 


\section{8}

Actividades para la implementación de la primer fase del proceso agroturístico

Las actividades de la primera fase de implementación, relacionadas con las labores al interior del sistema de producción.

a. Adecuación de vías de acceso a los predios (identificación y señalizaciones).

b. Implementación y adaptación de instalaciones, para la atención del turista. En hospedaje convencional en habitaciones típicas y confortables o en carpas tipo camping.

c. Diversificación y diferenciación de productos, clasificándolos por no procesados y procesados o transformados (tabla 3 ).

Tabla 3. Relación de diversificación de productos para la implementación del proceso agroturístico de Ocaña.

\begin{tabular}{|c|c|}
\hline Producto & Descripción \\
\hline Venta de manuales o cartillas para la transformación de & Preparación de recetas con productos frescos \\
\hline Manuales (creación de competencias en los niños) & Manuales para la siembra y manejo de la buerta. \\
\hline Venta de productos artesanales y recuerdos (suvenires) & $\begin{array}{l}\text { Pulseras, collares, camisetas, pocillos, llaveros con el } \\
\log _{0} \text { o nombre del sistema de producción. }\end{array}$ \\
\hline $\begin{array}{l}\text { Comercialización de productos cultivados y producidos } \\
\text { en sistema de producción. }\end{array}$ & $\begin{array}{l}\text { Degustaciones y venta de alimentos, postres, cortados, } \\
\text { conservas, con base en las hortalizas producidas, leche, } \\
\text { quesos y embutidos. }\end{array}$ \\
\hline
\end{tabular}

d. Ajuste de recorridos y participación en actividades del sistema de producción.

e. Promover la realización de actividades lúdicas y festejos que promuevan los servicios que prestan los sistemas de producción.

f. Creación de un proceso de capacitación en temáticas como atención turística, gastronomía, manejo financiero y contable de los procesos.

g.Implementacióndeherramientas informáticas para publicitar los servicios através de la centralización de la información de las riqueza cultural, histórica y natural de esta región y proyectarla de manera organizada a turistas de todo el mundo haciendo difusión por medio de Internet [10].

Actividades para la implementación de la segunda fase del proceso agroturístico

De forma complementaria, se deben fortalecer y formalizar los recorridos turísticos (rutas agroturísticas) ofertadas de dos formas: activamente con recorridos directos del turista en zonas tradicionales. Esto incluye también, los recorridos por zonas de recursos naturales patrimoniales, sitios culturales e históricos. O pasivamente, con el uso de videos y conferencias (con temáticas históricas de la región y procesos agropecuarios). Por último, con la creación de aplicaciones informáticas (App's), que muestren los activos de los sistemas de producción y de las rutas complementarias [11].

\section{Conclusiones}

La variedad y particularidad de los procesos agropecuarios efectuados en los sistemas de producción del municipio de Ocaña, representaran nuevas oportunidades para el desarrollo económico de la región.

La culminación exitosa de la propuesta agroturística depende de la organización, voluntad política e interés en el desarrollo de emprendimientos, para la creación de "alianzas" entre el sector productivo y las Universidades para la búsqueda de respaldo gubernamental.

La infraestructura turística en el municipio de Ocaña, así como la formación en gastronomía, capacitación en manejo contable, servicio de atención al turista y estructura legal, son considerados como factores fundamentales y condicionantes para el desarrollo de la propuesta. 
Es recomendable la realización de estudios de mercado para fortalecer y validar a la factibilidad de la propuesta, para determinar con certeza el segmento de población que se identifique. Adicionalmente, se debe realizar estudios de impacto ambiental, para determinar el grado de sustentabilidad y la incidencia que producirá el incremento turístico en la región.

\section{Referencias}

[1] G. Velázquez Valadez y G.A Cardona Reséndiz, "El crecimiento económico en México y su relación con el turismo y medio ambiente (2003-2015)", Mundo Fesc, vol. 7, n. ' 13, pp. 56-72, sep. 2017

[2] C.A Muñóz Alvarez, R.C Osorio Mass, L.F Alfonso Garzón, H. Muñóz Hernández y J.D Canabal Guzmán, "Ecoturismo, marketing ecológico y postconflicto, una mirada en contexto para el departamento de Córdoba", Aglala, vol. 9, no. 1, pp. 91-12, 2018. https:// doi.org/10.22519/22157360.1183.

[3] Organización Mundial del Turismo. OMT. Seminario internacional sobre gestión de destinos: nuevos desafíos, nuevas soluciones., 2012, [En línea]. Disponible en https://bit. ly/2Z11r16

[4] J.L García Cuesta, "El Turismo Rural como factor diversificador de rentas en la tradicional economía agraria", Estudios Turísticos, $\mathrm{n}^{\circ}$. 132, pp. 45-60, 1996

[5] Sensación Rural, "Portal turístico. Agroturismo en España, ¡más allá del turismo rural!”, 2018. [En Línea]. Disponible en https://bit.ly/2JD8IST

[6] A. Gannon, "Rural tourism as a factor in rural community economic development for economies transition", Journal of Sustainable Tourism, vol. 2, no. 1-2, pp. 51-60, 1994

[7] J.P. Roig, "Turismo rural: cómo América Latina puede sacar más provecho", América económica, 2014. [En línea]. Disponible en: https://bit.ly/30GLAZd

[8] M. Leal, "Propuesta de turismo rural para la región andina". En: IICA, 2008, [En línea]. Disponible en https://bit.ly/2XNtV1Y

[9] Ley 1558 de 2012, "Nueva ley del turismo", Congreso de la República de Colombia., 2012. [En línea]. Disponible en https://bit. ly/2Y901HT.

[10] M. Pacheco, B. Arévalo Vera, y I. K. Rincón Parada, "La Web, estrategia para incentivar el turismo", Revista Ingenio, vol. 8, n. ${ }^{\circ}$ 1, pp. 123130, nov. 2015.

[11] G. Freiría, "El turismo rural como promotor del empleo y las microeconomías en el desarrollo territorial rural. En: Seminario sobre Turismo Rural y su Contribución a la Creación de Empleo y a la Conservación del Patrimonio Asunción.” OMT, 2003. [En línea]. Disponible en: https://bit.ly/2Sm2SYG. 